\title{
Leaf Rust Resistance of Spring, Facultative, and Winter Wheat Cultivars from China
}

\author{
R. P. Singh, International Maize and Wheat Improvement Center (CIMMYT), Lisboa 27, Apdo. Postal 6-641, \\ 06600, Mexico, D.F., Mexico; W. Q. Chen, Institute of Plant Protection, Chinese Academy of Agricultural Sci- \\ ences, West Yuan Ming Yuan Road, Beijing 100094, P.R. China; and Z. H. He, Liaison Scientist, CIMMYT \\ China office, C/O Chinese Academy of Agricultural Sciences, No. 30 Baishiqiao Rd., Beijing 100081, P.R. \\ China
}

\begin{abstract}
Singh, R. P., Chen, W. Q., and He, Z. H. 1999. Leaf rust resistance of spring, facultative, and winter wheat cultivars from China. Plant Dis. 83:644-651.

Leaf rust, caused by Puccinia triticina, is an important disease of wheat (Triticum aestivum) in China. Sixty-one spring and 102 facultative or winter growth habit wheat cultivars from China and a set of testers, carrying named $L r$ genes, were evaluated for resistance at the seedling growth stage with an array of Mexican Puccinia triticina races. Variation in seedling infection types of the cultivars was compared with that of the testers, and genes conferring low infection types were postulated. In total, nine named genes, Lrl (in 13 cultivars), Lr3 (12), Lr3bg (2), $\operatorname{Lr} 10$ (1), Lr13 (4), Lr14a (1), Lr16 (49), Lr23 (9), and Lr26 (81), were identified. Thirty-one cultivars displayed intermediate reactions to one or more races that could not be attributed to any named gene. Twenty-eight spring cultivars were also evaluated at two field sites in Mexico using two common races. About half of them displayed good to moderate adult resistance that may be partly due to the presence of slow rusting gene Lr34 in at least seven cultivars. Diversity in adult plant responses of these wheats indicated the presence of additional slow rusting genes. Presence of $1 \mathrm{~B} .1 \mathrm{R}$ translocation in 12 wheat cultivars, supposedly derived from intergeneric crosses involving T. durum, Haynaldia villosa, and Avena fatua, indicated that their pedigrees were incorrect.
\end{abstract}

Wheat (Triticum aestivum L.) is grown in China on about 29 million hectares in diverse environments between 25 and $50^{\circ} \mathrm{N}$ latitudes (5). Using 1988 statistics, spring wheat is the most common wheat type grown (47\%), planted mostly in autumn, but also in spring. Facultative wheat contributes about $40 \%$ of the wheat area in China, and winter wheat makes up the remaining $13 \%$ (4). Among several diseases that constrain production, leaf rust (caused by Puccinia triticina Eriks.) is probably the most widely distributed disease, as it affects winter, facultative, and spring wheats. Genetic resistance is the most economical and environmentally benign control measure. Chinese wheats from the Yangtze River Valley are currently being used all over the world as sources of scab (caused by Fusarium spp.) resistance (1). Little is known about the leaf rust genes present in these and other Chinese wheats.

Corresponding author: R. P. Singh

E-mail: r.singh@cgiar.org

Accepted for publication 11 March 1999.

Publication no. D-1999-0429-01R

(C) 1999 The American Phytopathological Society
The ability to diversify the genetic base for resistance depends on knowledge of resistance genes present in the germ plasm used in a breeding program. Named racespecific genes for resistance to rusts in wheat can be postulated if a wheat line is inoculated with a series of pathogen races that possess diverse combinations of avirulence and virulence genes. This method, which is based on the gene-forgene relationship, has been described and used by several researchers $(2,8,16,17,20,23)$. Postulations can be further confirmed, if necessary, by genetic analyses.

The objectives of our study were to determine if these Chinese wheats contain previously described genes that confer resistance at the seedling growth stage and to assess the presence of adult resistance in selected spring wheats.

\section{MATERIALS AND METHODS}

The 61 spring and 102 facultative or winter wheat cultivars tested are listed in Tables 1 and 2, respectively, along with the province where they originated, pedigree, and area sown during 1995. Seeds of these cultivars were provided by the Chinese Academy of Agricultural Sciences. A set of tester lines, mostly near-isogenic for known single genes in a Thatcher (CI 10003) background, was also included. Seed of the testers, originally provided by the Cereal Disease Laboratory, St. Paul, Minnesota, and the Plant Breeding Institute of the University of Sydney, Australia, are maintained at CIMMYT.

The nomenclature system for the 14 races of $P$. triticina (Table 3 ) used in the study follows the system of Long and Kolmer (9), with two additional supplementary sets (14). Vacuum-dried (12) urediniospores of these races are stored at CIMMYT. Fresh inoculum was obtained by multiplying urediniospores on the susceptible cultivar Morocco.

Fully expanded primary leaves of 9-dayold seedlings of cultivars and testers were inoculated by spraying urediniospores suspended in the light-weight mineral oil Soltrol 170 (Phillips 66 Company, Oklahoma) at a concentration of 2 to $3 \mathrm{mg} / \mathrm{ml}$. Different sets of seedlings were used for inoculation with each of the 14 races, and six to eight seedlings planted in a clump were used in each test. In addition to the above tests, two sets of 14-day-old seedlings of cultivars and testers were also inoculated with races $\mathrm{LCJ} / \mathrm{BN}$ and $\mathrm{NCJ} / \mathrm{BN}$. Inoculated plants were placed in a dark dew chamber overnight at 18 to $20^{\circ} \mathrm{C}$, dried for an additional $4 \mathrm{~h}$ under light, and transferred to a greenhouse maintained at 20 to $25^{\circ} \mathrm{C}$. Infection types were recorded after 9 to 12 days using a 0 to 4 scale for leaf rust similar to that described by Stakman et al. (22) for stem rust. Tests were repeated twice during different months. Infection type data from only one test are reported because of the lack of significant variation in infection types in the two tests.

The presence of leaf rust resistance genes ( $L r$ genes) in seedlings of the cultivars was postulated by comparing the low and high infection types displayed by the cultivars with the infection type of known $L r$ genes in the testers (20).

Field evaluations of the spring wheat cultivars for resistance to leaf rust were carried out in Mexico at CIMMYT's research stations at Ciudad Obregon (Sonora State) and El Batan (Mexico State) during the 1992-93 and 1993 crop cycles, respectively. Both locations have environments conducive to leaf rust development. Plots consisted of two 1-m rows seeded $20 \mathrm{~cm}$ apart, with $70 \mathrm{~cm}$ 
between plots and a path of $50 \mathrm{~cm}$. Each plot consisted of approximately 30 to 40 plants. The experiment was planted twice at both locations in isolated areas. In the first experiment, the leaf rust epidemic initiated with race TCB/TD spread in the field from inoculated plants of the sus- ceptible cultivar Morocco (PI 431591) sown as clumps at one end of each plot in the path. Spreader rows of cultivar Jupateco 73S, inoculated with race TBD/TM, were used in the second experiment. Jupateco $73 \mathrm{~S}$ is highly susceptible to race $\mathrm{TBD} / \mathrm{TM}$ but not to
TCB/TD. The two races were common field races in Mexico during the period when the experiments were carried out.

Leaf rust severity and host response data on flag leaves were recorded at growth stages 85 to 87 (soft-dough to hard-dough) on the Zadoks' scale (24). Severity estima-

Table 1. Origin, pedigree, and cultivated area of 61 Chinese spring wheat cultivars tested for resistance to Puccinia triticina

\begin{tabular}{|c|c|c|c|c|}
\hline No. & Name & $\begin{array}{l}\text { Originating } \\
\text { province }\end{array}$ & Pedigree & $\begin{array}{l}\text { Cultivated } \\
\text { in } 1995 \text { (ha) }\end{array}$ \\
\hline 1 & Emai 9 & Hubei & Emai 6 Sel. & 92,666 \\
\hline 2 & Huazong 6 & Hubei & Mentana/T. turgidum & $\ldots{ }^{b}$ \\
\hline 3 & Wannian 2 & Jiangxi & Mentana sel. & $\ldots$ \\
\hline 4 & Yangmai 3 & Jiangsu & Yangmai 1 sel. & $\ldots$ \\
\hline 5 & Yangmai $85-128$ & Jiangsu & Yangmai 3/Chuan 74-4787 & Not released \\
\hline 6 & Sumai 3 & Jiangsu & Funo/Taiwan wheat & $\ldots$ \\
\hline 7 & Annong 8903 & Anhui & Bainong 3217/Annong 2//St 2422/464 & $\ldots$ \\
\hline 8 & Mianyang 80-8 & Sichuan & Fan 6 sel. & $\ldots$ \\
\hline 9 & Chuangyu 5 & Sichuan & $67-1776 / 6604$ & $\ldots$ \\
\hline 10 & Chuanmai 22 & Sichuan & Mianyang 11/Chuanmai 20 & $\ldots$ \\
\hline 11 & Guinong 26 & Guizhou & Haynaldia villosa/Avena fatual/T. aestivum & $\ldots$ \\
\hline 12 & Xuzhou 21 & Jiangsu & Punong 3665/UP301 & $\ldots$ \\
\hline 13 & Ningxia 216 & Ningxia & $\ldots$ & $\ldots$ \\
\hline 14 & Ningxia 86Y1608 & Ningxia & $\ldots$ & $\ldots$ \\
\hline 15 & Kefeng 3 & Heilongjiang & Ke 71 F4-370-7/Mexipak 66 & $\ldots$ \\
\hline 16 & Yangmai 4 & Jiangsu & Mentana/Triumph//Funo sel. & 58,667 \\
\hline 17 & Yangmai 5 & Jiangsu & Mentana/Triumph//Funo/3/St 1472/506 & 984,000 \\
\hline 18 & Yangmai 85-46 & Jaingsu & Chuan 74-324/Zaohong & Not released \\
\hline 19 & Yangmai 85-85 & Jiangsu & $\mathrm{Fu} 728 / 9-6-1-2$ & $\ldots$ \\
\hline 20 & Yangmai 87-158 & Jiangsu & Mentana/Triumph//Funo/3/St 1472/506 & 405,333 \\
\hline 21 & $\mathrm{Su} 8060$ & Jiangsu & Sufan 411/Wanya 098 & $\ldots$ \\
\hline 22 & Ning 8675 & Jiangsu & 80D492/Yangmai 3 & $\ldots$ \\
\hline 23 & Fan 6 & Sichuan & $\begin{array}{l}\text { IBO 1828/NP 824/3/Wuyimai//Chengdu Guangtou/Zhongnong } \\
\text { 28/4/Zhongnong 28/ IBO 1828//NP 824/Funo }\end{array}$ & $\cdots$ \\
\hline 24 & Mianyang 11 & Sichuan & Airongsui/Ya anzao//Fan 6 & $\ldots$ \\
\hline 25 & Chuanyu 10 & Sichuan & Kavkaz/983//980/3130 & $\ldots$ \\
\hline 26 & Kefeng 5 & Heilongjiang & $\ldots$ & $\ldots$ \\
\hline 27 & Kehan 10 & Heilongjiang & Ke 86-88/Ke 68-585-13//T808/Ke69-513 & 84,667 \\
\hline 28 & Shanghai 3 & Shanghai & $\ldots$ & $\ldots$ \\
\hline 29 & Shanghai 5 & Shanghai & $\ldots$ & $\ldots$ \\
\hline 30 & Guinong 22 & Guizhou & Haynaldia villosa/Sauwene 20 & $\ldots$ \\
\hline 31 & Wanxie 1128 & Anhui & Lumai 1/Xian Aitezao//Guinong 21 & $\ldots$ \\
\hline 32 & Zhejiang 4 & Zhejiang & $\ldots$ & $\ldots$ \\
\hline 33 & Yumai 5 & Henan & Funo/Zhengzhou 6//Zhengzhou 6609 & $\ldots$ \\
\hline 34 & Yumai 7 & Henan & Predgornaia 2/Yanshi 4 & $\ldots$ \\
\hline 35 & Yumai 15 & Henan & Yanshi 4/Panjiang 3 & $\ldots$ \\
\hline 36 & Guinong 93-9c & Guizhou & T. durum/Haynaldia villosa//Avena fatua & Not released \\
\hline 37 & Chuanyu 11 & Sichuan & $\ldots$ & $\ldots$ \\
\hline 38 & Guinong 93-3c & Guizhou & T. durum/Haynaldia villosa//Avena fatua & Not released \\
\hline 39 & Guinong $93-5^{\mathrm{c}}$ & Guizhou & T. durum/Haynaldia villosa//Avena fatua & Not released \\
\hline 40 & Guinong $93-7^{\mathrm{c}}$ & Guizhou & T. durum/Haynaldia villosa//Avena fatua & Not released \\
\hline 41 & Chuanmai 18 & Sichuan & $69-1776 / 663$ & $\ldots$ \\
\hline 42 & Chuanmai 21 & Sichuan & Mianyang 11//983/Kavkaz & $\ldots$ \\
\hline 43 & Emai 1 & Hubei & Mentana sel. & $\ldots$ \\
\hline 44 & Een 1 & Hubei & Lovrin 10/Zhengzhou 761//Sumai 3 & 545,333 \\
\hline 45 & Fujing 5114 & Fujian & $\ldots$ & $\ldots$ \\
\hline 46 & Wuhan 3 & Wuhan & $\ldots$ & $\ldots$ \\
\hline 47 & Zhengzhou 871 & Henan & Zhengzhou 761/Wumang 77 & $\ldots$ \\
\hline 48 & Zhengzhou 872 & Henan & Zhengzhou 761/Wumang 77 & $\ldots$ \\
\hline 49 & $10-\mathrm{A}$ & Sichuan & Ya'anzao/Oinling Secale//Aurora/3/88-1643 & $\ldots$ \\
\hline 50 & Mianyang 26 & Sichuan & Mianyang 20/Chuanyu 9 & $\ldots$ \\
\hline 51 & Guinong 20-92004c & Guizhou & T. durum/Haynaldia villosa & Not released \\
\hline 52 & Guinong 20-92008c & Guizhou & T. durum/Haynaldia villosa & Not released \\
\hline 53 & Guinong 20-92011 ${ }^{\mathrm{c}}$ & Guizhou & T. durum/Haynaldia villosa & Not released \\
\hline 54 & Guinong 20-92013c & Guizhou & T. durum/Haynaldia villosa & Not released \\
\hline 55 & Guinong 20-92014c & Guizhou & T. durum/Haynaldia villosa & Not released \\
\hline 56 & Guinong 20-92017c & Guizhou & T. durum/Haynaldia villosa & Not released \\
\hline 57 & Guinong 20-92019c & Guizhou & T. durum/Haynaldia villosa & Not released \\
\hline 58 & Guinong 20-92002c & Guizhou & T. durum/Haynaldia villosa & Not released \\
\hline 59 & New Kehan 9 & Heilongjiang & Kefeng $2 / \mathrm{Ke} 74$ F3-249-3 & 464,667 \\
\hline 60 & Chuanyu 8 & Sichuan & Ar'ai/4/Fan 6/3/Fan 6/Yuan 110//170-8 & 8,000 \\
\hline 61 & Chuanyu 12 & Sichuan & Chuanyu $8 / 4516$ & $\ldots$ \\
\hline
\end{tabular}

${ }^{a}$ Source: Ministry of Agriculture, P.R. China.

${ }^{b}$ Data on pedigree or cultivated area not available.

${ }^{\mathrm{c}}$ Pedigree given to authors is incorrect based on the presence of the $1 \mathrm{~B} .1 \mathrm{R}$ translocation in the cultivar. 
tions were based on the modified Cobb scale (11), and the host response to infection was evaluated following Roelfs et al. (12).

\section{RESULTS AND DISCUSSION}

Variation in infection types (ITs) of the known $L r$ gene carrying tester lines, inoculated with $14 P$. triticina races (Table 3 ), indicated possibility of the identification of 19 resistance genes $(L r l, 2 a, 2 b, 2 c$, $3,3 b g, 10,11,13,14 a, 15,16,17,19,23$, $24,26,27+31$, and 28). Resistance genes
Lr3ka, 9, 21, 25, 29, 30, 32, 33, and 35 displayed low or intermediate ITs with all races. Absence of an $\mathrm{Lr}$ gene is indicated in a cultivar whenever a high IT is observed with any of the races used in the tests. Postulation of genes $\operatorname{Lr} 12,14 b, 18$, $20,22 a, 22 b$, and 35 was not possible with these 14 races because high ITs were recorded with each race. Gene $\operatorname{Lr} 18$ can be detected with Mexican races only if tested at temperatures below $18^{\circ} \mathrm{C}$ (14). Similarly, Lr34 can give low ITs at low temperatures combined with low light intensi- ties. Genes $L r 12,22 a, 22 b$, and 35 are known to be effective only in adult plants (10).

Eleven spring wheat cultivars (numbered 1-11, Table 4) and 18 facultative or winter wheat cultivars (numbered 1-18, Table 5) displayed high ITs with all races; therefore, we could not postulate any known $L r$ gene in these cultivars.

The spring wheat cultivar Xuzhou 21 (numbered 12, Table 4) and four facultative/winter wheat cultivars (numbered 1922, Table 5) displayed IT 0; with the $\operatorname{Lrl}$

Table 2. Origin, pedigree and cultivated area of 102 Chinese facultative or winter wheat cultivars, tested for resistance to Puccinia triticina

\begin{tabular}{|c|c|c|c|c|}
\hline No. & Name & $\begin{array}{c}\text { Originating } \\
\text { province }\end{array}$ & Pedigree & $\begin{array}{c}\text { Cultivated } \\
\text { in } 1995 \text { (ha) }\end{array}$ \\
\hline 1 & Xiaoyan 107 & Shaanxi & Mianyang 75-20/Xiaoyan 392 & $\ldots \mathrm{b}$ \\
\hline 2 & Shaanmai 897 & Shaanxi & Xiaoyanmai (Zhong 4)/3811(2) & 94,667 \\
\hline 3 & Changwu 132 & Shaanxi & $\ldots$ & $\ldots$ \\
\hline 4 & 81168 & Shaanxi & $\ldots$ & $\ldots$ \\
\hline 5 & Zhongliang 12 & Gansu & Chambord/Kangyin 655 & $\ldots$ \\
\hline 6 & Tianxuan 15 & Gansu & Jubileina 2/Abbondanza & $\ldots$ \\
\hline 7 & Tianxuan 40 & Gansu & Abbondanza/Jaral//691776 & $\ldots$ \\
\hline 8 & Tianxuan 871 & Gansu & $\ldots$ & $\ldots$ \\
\hline 9 & Neixiang 5 & Henan & Mentana/(Baihuomai+Quality+Baimangmai) & $\ldots$ \\
\hline 10 & Pingyuan 50 & Henan & Local cultivar & $\ldots$ \\
\hline 11 & Youzimai & Henan & Local cultivar & $\ldots$ \\
\hline 12 & Zhengzhou 5389 & Henan & Local cultivar & $\ldots$ \\
\hline 13 & Jinmai 16 & Shanxi & Gongnong 12/6014 & 35,333 \\
\hline 14 & Jinmai 31 & Shanxi & Shaan 6811/Linfen 5610 & 124,667 \\
\hline 15 & Ji86-5 & Hebei & Lovrin 10/Aiganzao & Not released \\
\hline 16 & Yannong 15 & Shandong & Youxuan 57//St2422/464 & 192,667 \\
\hline 17 & Taigang 113 & Shandong & Taishan 2/Yannong 15//Jinghua 1 & $\ldots$ \\
\hline 18 & Jing 411 & Beijing & Fengkang 2/74 Chang 1 & 197,333 \\
\hline 19 & Xuzhou 23 & Jiangsu & Punong 3665/UP301//Zhengzhou 761 & $\ldots$ \\
\hline 20 & Wenxian 4 & Henan & Wenxian 2540 Sel. & 41,333 \\
\hline 21 & Jimai 31 & Hebei & Primepi/Inia 66//Taishan 5 & 59,333 \\
\hline 22 & Baiyoubao & Shandong & Youzhimai/Baoda 300 pao & $\ldots$ \\
\hline 23 & Bumai 90 & Jiangsu & Zhongmai 144 Sel. & $\ldots$ \\
\hline 24 & Xian 8 & Shaanxi & Aiganzao//St 1472/507 & 416,000 \\
\hline 25 & Jing 6226 & Beijing & $\ldots$ & $\ldots$ \\
\hline 26 & Xiaoyan 6 & Shaanxi & St 2422/464//Xiaoyan 96 & 242,000 \\
\hline 27 & Shaan 229 & Shaanxi & $7853 / 80256$ & 244,000 \\
\hline 28 & Shaan 611 & Shaanxi & (Zhong 3+Zhong 4)/T. aestivum & $\ldots$ \\
\hline 29 & Shaan 861 & Shaanxi & 1900-5-4-0-9-5/Xiaoyan 6 & $\ldots$ \\
\hline 30 & N82(2)-10 & Shaanxi & $9615-11 / 7014 \mathrm{RO}$ & $\ldots$ \\
\hline 31 & D10-2 & Shaanxi & $\ldots$ & $\ldots$ \\
\hline 32 & Tianshui 873 & Gansu & $\ldots$ & $\ldots$ \\
\hline 33 & Yumai 18 & Henan & Zhengzhou 761/Yanshi 4 & $1,396,667$ \\
\hline 34 & Ji 83-5180-10 & Hebei & $\ldots$ & $\ldots$ \\
\hline 35 & Jinmai 5 & Shanxi & BAU 16/Huabei 187 & $\ldots$ \\
\hline 36 & Jinmai 21 & Shanxi & 687-44/Predgornaia 2 & $\ldots$ \\
\hline 37 & Jinmai 23 & Shanxi & 68G44F2/Ray & $\ldots$ \\
\hline 38 & Jimai 24 & Hebei & Anyang 10/Aifeng//Lovrin 10/70114 & 112,667 \\
\hline 39 & Jimai 30 & Hebei & 78-3147/Shi 4414 & 62,000 \\
\hline 40 & Ji 8454 & Hebei & $\ldots$ & $\ldots$ \\
\hline 41 & Ji 87-4314 & Hebei & Yun $78-4 / / 78-3662 / 78-4101$ & $\ldots$ \\
\hline 42 & Ji 874-109 & Hebei & Yunzhao 1/4001 & Not released \\
\hline 43 & Yumai 17 & Henan & Yanda 7406/Nanyang 75-6 & 131,333 \\
\hline 44 & Yumai 21 & Henan & Bainong 791/Yumai 2//Lumai 1/Yanshi 4 & 90,667 \\
\hline 45 & Yanshi 86117 & Henan & Qianfeng 1/3/Predgornaia 2//Yanshi 4/Xiaoyan 5 & $\ldots$ \\
\hline 46 & Lumai 1 & Shandong & Aifeng 3//Mengxian 201/Neuzucht & 406,667 \\
\hline 47 & Lumai 11 & Shandong & $\ldots$ & $\ldots$ \\
\hline 48 & Lu 215953 & Shandong & Aimengniu/Fu 66 & $\ldots$ \\
\hline 49 & Lumai 14 & Shandong & $\mathrm{C} 149 / \mathrm{F} 4530$ & $1,000,000$ \\
\hline 50 & Yan $7578-128$ & Shandong & Lovrin 10/3/Virgilio/Rulofen//Youbao & $\ldots$ \\
\hline 51 & Jimai 23 & Hebei & Anyang 10/Aifeng 1//Lovrin 10/70114 & $\ldots$ \\
\hline 52 & Fengkang 2 & Beijing & Youmangbai 4/Lovrin 10 & $\ldots$ \\
\hline 53 & Fengkang 8 & Beijing & Youmanghong/Lovrin 10 & 98,667 \\
\hline \multirow[t]{2}{*}{54} & Jingdong 1 & Beijing & Jinzhong 849/F49-70//Youmanghong 7 & $\ldots$ \\
\hline & & & & (continued on 1 \\
\hline
\end{tabular}

\footnotetext{
a Source: Ministry of Agriculture, P.R. China.

${ }^{\mathrm{b}}$ Data on pedigree or cultivated area not available.
} 
avirulent races $\mathrm{BBB} / \mathrm{BN}, \mathrm{CBJ} / \mathrm{QB}$, $\mathrm{CBJ} / \mathrm{QL}, \mathrm{CBJ} / \mathrm{QQ}$, and CCJ/SP and high reaction with the remaining nine races. Because this IT pattern matches that of the Lrl-carrying tester (Table 3), we postulate the presence of $\mathrm{Lrl}$ in these cultivars. Indian wheat cultivar UP301, one of the progenitors of Xuzhou 21 and Xuzhou 23, is known to carry gene $\mathrm{Lrl}$ (17).

Ningxia 216 and Ningxia 86Y1608 are likely to carry gene $\mathrm{Lr} 3 \mathrm{bg}$ because they displayed low IT; only with the three races avirulent to $L r 3 b g, \mathrm{BBB} / \mathrm{BB}$, $\mathrm{LCJ} / \mathrm{BN}$, and NCJ/BN (Table 4). However, it is difficult to be sure because Lr3 may cause a similar reaction, although the tester line for Lr3 displayed intermediate reactions with a few additional races.

Gene Lr10 may be present in the spring wheat Kefeng 3, seedlings of which displayed low ITs only with the four races that lacked virulence for $\operatorname{LrlO}$ (Table 4). Similarly, Lrl3 may be present in Bumai 90 and Xian 8 because mesothetic ITs X or $\mathrm{XX}+$ were observed in 9- or 14-day-old seedlings inoculated with $L r 13$-avirulent races $\mathrm{BBB} / \mathrm{BN}, \mathrm{LCJ} / \mathrm{BN}$, and $\mathrm{NCJ} / \mathrm{BN}$ (Table 5). Gene $L r 14 a$ could be present in Jing 6226; however, this postulation will require further verification because $\mathrm{BBB} /$ $\mathrm{BB}$ was the only avirulent race available for testing, and the resistance ITs displayed by the $L r 14 a$ tester line and Jing 6226 were barely intermediate (Tables 3 and 5).
Gene Lrl6 appears to be present alone in numerous spring (numbered 16-31, Table 4) and facultative or winter wheats (numbered 26-34, Table 5). These wheats displayed ITs ranging from 1 to $3 \mathrm{c}$, similar to the Lrl6 tester, with all races. Similarly, gene $L r 26$ was present alone in five spring (numbered 32-36, Table 4) and 27 facultative and winter wheat cultivars (numbered 35-61, Table 5), as evidenced by their low ITs of 0 ; or ; with races $\mathrm{CBJ} / \mathrm{QL}, \mathrm{CBJ} / \mathrm{QQ}$, $\mathrm{TBB} / \mathrm{JP}$, and TBD/TM; ITs $; 1^{-}$to $13 \mathrm{c}$ with $\mathrm{BBB} / \mathrm{BB}$ and $\mathrm{CBJ} / \mathrm{QB}$; and high ITs with the remaining 10 races. Since gene $\operatorname{Lr} 26$ is known to be present in the $1 \mathrm{~B} .1 \mathrm{R}$ chromosome translocation (10), the presence of this translocation in these cultivars can be inferred. The origin of this translocation in

Table 2. (continued)

\begin{tabular}{|c|c|c|c|c|}
\hline No. & Name & $\begin{array}{l}\text { Originating } \\
\text { province }\end{array}$ & Pedigree & $\begin{array}{c}\text { Cultivated } \\
\text { in } 1995 \text { (ha) }^{\mathrm{a}}\end{array}$ \\
\hline 55 & Dongxie 3 & Beijing & Jingshuang 2/Predgornaia 2//Jingshuang 3 & $\ldots$ \\
\hline 56 & Bau 146 & Beijing & $\ldots$ & $\ldots$ \\
\hline 57 & Qinmai 6 & Shaanxi & $\ldots$ & $\ldots$ \\
\hline 58 & 6010 & Shanxi & Bezostaja 1/72-6051//256-113 & $\ldots$ \\
\hline 59 & Zhongliang 7553 & Gansu & Nongda 181/Lovrin 13 & $\ldots$ \\
\hline 60 & Huaiyin 9554 & Jiangsu & Yumai 13/Yan 1604 & $\ldots$ \\
\hline 61 & Lifen 7203 & Shanxi & Saric/3029/3/74100//Youbao 036/Xiaoyan 759 & 20,000 \\
\hline 62 & Tianshui 872 & Gansu & & \\
\hline 63 & Yumai 13 & Henan & Bainong 3217/9612-2 & 44,667 \\
\hline 64 & Bainong 3217 & Henan & Funo/Neixiang 5//Xiannong 39/3/64(4)3/Yanda 24 & 17,333 \\
\hline 65 & Bainong 64 & Henan & $\begin{array}{l}\text { Bainong 8717/3/Yanda 72-629-52/Shi 82-55941 } \\
\text { //Bainong 84-4046-1 }\end{array}$ & $\ldots$ \\
\hline 66 & Ji 89-6232 & Hebei & Bainong 3217/Dongxie 2 & $\ldots$ \\
\hline 67 & Jinghua 5 & Beijing & Aurora/Beijing 15//Youmanghong 7 & $\ldots$ \\
\hline 68 & Siyang 128 & Jiangsu & Siyang 79-23/Siyang 1883 & $\ldots$ \\
\hline 69 & Kangyin 655 & Shanxi & Beijing 8/Suwon 11/Skorospelka 3 & \\
\hline 70 & Shaan 213 & Shanxi & TJB/7336 & 13,333 \\
\hline 71 & Shaan 7852 & Shanxi & $\ldots$ & \\
\hline 72 & Shaan 7859 & Shanxi & $\begin{array}{l}\text { Predgornaia 2/66-46-18/3/Sibley } 81 / / \\
\text { Fengkang 3/62(9)2-1 }\end{array}$ & 205,333 \\
\hline 73 & Xuzhou 174 & Jiangsu & Sichuan uni-culm wheat/Predgornaia 2//Punong 3665 & $\ldots$ \\
\hline 74 & Huaiying 894-2 & Jiangsu & Yan C 172/Gao 38 White//Qianfeng 1 & $\ldots$ \\
\hline 75 & Lumai 15 & Shandong & Tal Yangmai 1/757318//104-4 & 849,333 \\
\hline 76 & Ji 84-5103 & Hebei & Xiaoyan 7014-5-2-14/78-4101 & $\ldots$ \\
\hline 77 & N82(2)-18 & Shanxi & 9615-11/7014R0 & $\ldots$ \\
\hline 78 & Beijing 837 & Beijing & $\begin{array}{l}\text { Youmanghong } 7 / \text { Lovrin } 10 / 3 / \text { Jingshuang } 6 / \\
\text { Predgornaia } 2 / 2 / \text { Jingshuang } 3\end{array}$ & $\cdots$ \\
\hline 79 & Beijing 841 & Beijing & Beijing 18/Fengkang 4/Nongda 139 & 34,000 \\
\hline 80 & BAU 146 & Beijing & Chi5021/Rusalka//7201/Lovrin 13 & $\ldots$ \\
\hline 81 & CA 8646 & Beijing & Fengkang 4//Shi 5-061/Youmangbai-Lovrin 10 & $\ldots$ \\
\hline 82 & Yumai 16 & Henan & Zhengzhou 761//Baimian 3/Kavkaz & 56,000 \\
\hline 83 & Lumai 14 & Shandong & 74(113)/3/Yan 70172/Yan 71152//Yan 71148 & 855,333 \\
\hline 84 & Jin 2148 & Fujian & Jinjiangchizhi/Huadong 5//Orofen/3/Rieti 11 & 18,666 \\
\hline 85 & Wan 8550 & Anhui & T. monососсит//Aurora/Ray/3/NPFP 5443/3 & $\ldots$ \\
\hline 86 & Yan 2419 & Shandong & $7222-321 / 74(11)$ & $\ldots$ \\
\hline 87 & Shaan 167-6 & Shanxi & TA $174-33 / 2587$ & \\
\hline 88 & Zhoumai 10 & Henan & Mianyang $81-5 / 78 \mathrm{~A}$ & 185,333 \\
\hline 89 & Lantian 1 & Gansu & $\ldots$ & $\ldots$ \\
\hline 90 & Shaan 7554 & Shanxi & $\ldots$ & $\ldots$ \\
\hline 91 & Laizhoujian 1-8 & Shandong & $\ldots$ & $\ldots$ \\
\hline 92 & Laizhou 953 & Shandong & Skorospelka 5/Yexun 1//7832110-1 & 248,000 \\
\hline 93 & Huaiyin 86220 & Jiangsu & Yan C172/Gao 38 White//Qianfeng 1/3/Shaan 7859 & $\ldots$ \\
\hline 94 & Yumai 2 & Henan & 65(14)3/Huixianhong & 254,000 \\
\hline 95 & Zhengzhou 8329 & Henan & $78(6) 9-2 / 80(6)-6-10$ & 36,000 \\
\hline 96 & Beijing 10 & Beijing & Hubei 672/Xinshi 14//Skorospelka 1B/Huabei 672 & $\ldots$ \\
\hline 97 & Shaan 897 & Shanxi & Xiaoyanmai (Zhong 4)/3811(2) & $\ldots$ \\
\hline 98 & Wanmai 19 & Anhui & Bao 7422/Yumai 2 & $\ldots$ \\
\hline 99 & Xiannong 4 & Shanxi & Local cultivar & $\ldots$ \\
\hline 100 & Mazhamai & Shanxi & Local cultivar & $\ldots$ \\
\hline 101 & Zhong 4 & Shanxi & Keqiang/Mentana//Ag. intermedium & $\ldots$ \\
\hline 102 & Jinmai 33 & Shanxi & $\begin{array}{l}\text { Naixue/5027//036/3/76-1295/4/ } \\
\text { Weidong 7/Xiangyang } 4\end{array}$ & 268,000 \\
\hline
\end{tabular}


Table 3. Seedling infection type responses of testers with known $L r$ genes when inoculated with 14 races of Puccinia triticina

$P$. triticina race and infection type ${ }^{\mathrm{a}}$

\begin{tabular}{|c|c|c|c|c|c|c|c|c|c|c|c|c|c|c|c|c|c|}
\hline Tester & $L r$ gene & $\begin{array}{c}\mathbf{B B B} / \\
\mathbf{B B}\end{array}$ & $\begin{array}{c}\text { CBJ/ } \\
\text { QB }\end{array}$ & $\begin{array}{c}\text { CBJ/ } \\
\text { QL }\end{array}$ & $\begin{array}{c}\text { CBJ/ } \\
\text { QQ }\end{array}$ & $\begin{array}{c}\text { CBJ/ } \\
\text { SP }\end{array}$ & $\begin{array}{l}\text { LCJ/ } \\
\text { BN }^{\mathbf{b}}\end{array}$ & $\begin{array}{l}\text { LCJ/ } \\
\text { BNc }^{c}\end{array}$ & $\begin{array}{c}\text { NCJ/ } \\
\text { BN }^{\mathbf{b}}\end{array}$ & $\begin{array}{c}\text { NCJ/ } \\
\text { BN }^{c}\end{array}$ & $\begin{array}{c}\text { TBB/ } \\
\text { JP }\end{array}$ & $\begin{array}{c}\text { TBD/ } \\
\text { TM }\end{array}$ & $\begin{array}{c}\text { TCB/ } \\
\text { TB }\end{array}$ & $\begin{array}{c}\text { TCB/ } \\
\text { TD }\end{array}$ & $\begin{array}{c}\mathrm{MFB} / \\
\text { SP }\end{array}$ & $\begin{array}{c}\text { MCJ/ } \\
\mathbf{Q M}\end{array}$ & $\begin{array}{c}\text { MCJ/ } \\
\text { SP }\end{array}$ \\
\hline RL6003 & 1 & 0 & 0 & 0 & 0 & 0 & $\mathrm{H}^{\mathrm{d}}$ & $\mathrm{H}$ & $\mathrm{H}$ & $\mathrm{H}$ & $\mathrm{H}$ & $\mathrm{H}$ & $\mathrm{H}$ & $\mathrm{H}$ & $\mathrm{H}$ & $\mathrm{H}$ & $\mathrm{H}$ \\
\hline RL6016 & $2 a$ & ; & $;$ & ; & 0 & 0 & $; 1$ & $; 1$ & $; 1$ & 1 & $\mathrm{H}$ & $\mathrm{H}$ & $\mathrm{H}$ & $\mathrm{H}$ & ; & ; & ; \\
\hline RL6019 & $2 b$ & ; & $; 1^{-}$ & ; & ; & 0 & $1^{+}$ & $3 c$ & $11^{+}$ & $1+3 c$ & $\mathrm{H}$ & $\mathrm{H}$ & $\mathrm{H}$ & $\mathrm{H}$ & $; 1^{-}$ & ; & 0 \\
\hline RL6047 & $2 c$ & $1^{-}$ & $1^{+}$ & $; 1^{-}$ & $; 1^{-}$ & 0 & $3 \mathrm{c} 3$ & $\mathrm{H}$ & $\mathrm{H}$ & $\mathrm{H}$ & $\mathrm{H}$ & $\mathrm{H}$ & $\mathrm{H}$ & $\mathrm{H}$ & $; 1^{-}$ & ; & ; \\
\hline RL6002 & 3 & 1 & $23 c$ & $3 c 3$ & $3^{-}$ & $23 c$ & $; 1^{-}$ & ;12 & $; 1^{-}$ & ;1 & $\mathrm{H}$ & $\mathrm{H}$ & $\mathrm{H}$ & $\mathrm{H}$ & $\mathrm{H}$ & $3 c 3$ & $3^{-3}$ \\
\hline RL6007 & $3 k a$ & 12 & 12 & $; 2^{-}$ & $12^{-}$ & 12 & 12 & 12 & 12 & ; 12 & 12 & $12^{-}$ & $12^{-}$ & 12 & $12^{-}$ & ;12 & 12 \\
\hline RL6042 & $3 b g$ & $; 1^{-}$ & $\mathrm{H}$ & $\mathrm{H}$ & $\mathrm{H}$ & $\mathrm{H}$ & ; & $; 1$ & ; & $; 1^{-}$ & $\mathrm{H}$ & $\mathrm{H}$ & $\mathrm{H}$ & $\mathrm{H}$ & $\mathrm{H}$ & $\mathrm{H}$ & $\mathrm{H}$ \\
\hline RL6010 & 9 & 0 & 0 & ; & ; & ; & 0 & 0 & 0 & 0 & 0 & 0 & 0 & 0 & 0 & ; & 0 \\
\hline RL6004 & 10 & $1^{-}$ & ; & $\stackrel{\vec{H}}{\mathrm{H}}$ & $\vec{H}$ & $\vec{H}$ & $\mathrm{H}$ & $\mathrm{H}$ & $\mathrm{H}$ & $\mathrm{H}$ & $\mathrm{H}$ & $\mathrm{H}$ & ;1 & ;1 & $\mathrm{H}$ & $\vec{H}$ & $\mathrm{H}$ \\
\hline Hussar & 11 & $1+3$ & $\vec{H}$ & $\mathrm{H}$ & $\mathrm{H}$ & $\mathrm{H}$ & $\mathrm{H}$ & $\mathrm{H}$ & $\mathrm{H}$ & $\mathrm{H}$ & $3 c$ & $3 c$ & $3 c 3$ & $3 c 3$ & $3 \mathrm{c} 3$ & $\mathrm{H}$ & $\mathrm{H}$ \\
\hline RL6011 & 12 & $\mathrm{H}$ & $\mathrm{H}$ & $\mathrm{H}$ & $\mathrm{H}$ & $\mathrm{H}$ & $\mathrm{H}$ & $\mathrm{H}$ & $\mathrm{H}$ & $\mathrm{H}$ & $\mathrm{H}$ & $\mathrm{H}$ & $\mathrm{H}$ & $\mathrm{H}$ & $\mathrm{H}$ & $\mathrm{H}$ & $\mathrm{H}$ \\
\hline Manitou & 13 & $\mathrm{X}^{+}$ & $\mathrm{H}$ & $\mathrm{H}$ & $\mathrm{H}$ & $\mathrm{H}$ & $\mathrm{X}^{+}$ & $X$ & $\mathrm{X}^{+}$ & $X$ & $\mathrm{H}$ & $\mathrm{H}$ & $\mathrm{H}$ & $\mathrm{H}$ & $\mathrm{H}$ & $\mathrm{H}$ & $\mathrm{H}$ \\
\hline RL6013 & $14 a$ & $3 c$ & $\mathrm{H}$ & $\mathrm{H}$ & $\mathrm{H}$ & $\mathrm{H}$ & $\mathrm{H}$ & $\mathrm{H}$ & $\mathrm{H}$ & $\mathrm{H}$ & $\mathrm{H}$ & $\mathrm{H}$ & $\mathrm{H}$ & $\mathrm{H}$ & $\mathrm{H}$ & $\mathrm{H}$ & $\mathrm{H}$ \\
\hline RL6006 & $14 b$ & $\mathrm{H}$ & $\mathrm{H}$ & $\mathrm{H}$ & $\mathrm{H}$ & $\mathrm{H}$ & $\mathrm{H}$ & $\mathrm{H}$ & $\mathrm{H}$ & $\mathrm{H}$ & $\mathrm{H}$ & $\mathrm{H}$ & $\mathrm{H}$ & $\mathrm{H}$ & $\mathrm{H}$ & $\mathrm{H}$ & $\mathrm{H}$ \\
\hline RL6052 & 15 & $; 1^{-}$ & ; & ; & ; & $\mathrm{H}$ & $; 1^{-}$ & $; 1^{-}$ & $1^{-}$ & 1 & $\mathrm{H}$ & $\mathrm{H}$ & $\mathrm{H}$ & $\mathrm{H}$ & $\mathrm{H}$ & ; & $\mathrm{H}$ \\
\hline RL6005 & 16 & $3 \mathrm{c} 3$ & 1 & $1^{+}$ & $; 1^{-}$ & $1^{+} 3 \mathrm{c}$ & $1^{+}$ & 1 & $1^{+}$ & $1^{+} 3 \mathrm{c}$ & $1^{+}$ & 1 & $1^{+}$ & 1 & $1^{+}$ & $1^{+}$ & $1^{+}$ \\
\hline RL6008 & 17 & $; 1$ & $\mathrm{H}$ & $\mathrm{H}$ & $\mathrm{H}$ & $\mathrm{H}$ & $\mathrm{H}$ & $\mathrm{H}$ & $\mathrm{H}$ & $\mathrm{H}$ & $; 1^{-}$ & $\mathrm{H}$ & ;1 & $; 1^{-}$ & $; 1^{-}$ & $\mathrm{H}$ & $\mathrm{H}$ \\
\hline RL6009 & 18 & $22^{+}$ & $\mathrm{H}$ & $\mathrm{H}$ & $\mathrm{H}$ & $\mathrm{H}$ & $\mathrm{H}$ & $\mathrm{H}$ & $\mathrm{H}$ & $\mathrm{H}$ & $\mathrm{H}$ & $\mathrm{H}$ & $\mathrm{H}$ & $\mathrm{H}$ & $\mathrm{H}$ & $\mathrm{H}$ & $\mathrm{H}$ \\
\hline RL6040 & 19 & 0 & 0 & ; & $\mathrm{H}$ & 0 & 0 & 0 & 0 & 0 & 0 & 0 & 0 & 0 & 0 & 0 & 0 \\
\hline Thew & 20 & $\mathrm{H}$ & $\mathrm{H}$ & $\mathrm{H}$ & $\mathrm{H}$ & $\mathrm{H}$ & $\mathrm{H}$ & $\mathrm{H}$ & $\mathrm{H}$ & $\mathrm{H}$ & $\mathrm{H}$ & $\mathrm{H}$ & $\mathrm{H}$ & $\mathrm{H}$ & $\mathrm{H}$ & $\mathrm{H}$ & $\mathrm{H}$ \\
\hline RL6043 & 21 & $2^{+} 3 c$ & 12 & 12 & 12 & $3 c$ & 12 & $12^{+}$ & 12 & $22^{+}$ & 2 & 12 & 12 & 2 & 12 & 12 & $2^{+} 3$ \\
\hline RL6044 & $22 a$ & $\mathrm{H}$ & $\mathrm{H}$ & $\mathrm{H}$ & $\mathrm{H}$ & $\mathrm{H}$ & $\mathrm{H}$ & $\mathrm{H}$ & $\mathrm{H}$ & $\mathrm{H}$ & $\mathrm{H}$ & $\mathrm{H}$ & $\mathrm{H}$ & $\mathrm{H}$ & $\mathrm{H}$ & $\mathrm{H}$ & $\mathrm{H}$ \\
\hline Thatcher & $22 b$ & $\mathrm{H}$ & $\mathrm{H}$ & $\mathrm{H}$ & $\mathrm{H}$ & $\mathrm{H}$ & $\mathrm{H}$ & $\mathrm{H}$ & $\mathrm{H}$ & $\mathrm{H}$ & $\mathrm{H}$ & $\mathrm{H}$ & $\mathrm{H}$ & $\mathrm{H}$ & $\mathrm{H}$ & $\mathrm{H}$ & $\mathrm{H}$ \\
\hline RL6012 & 23 & 1 & $; 1^{-}$ & $; 1^{-}$ & 1 & $\mathrm{H}$ & $\mathrm{H}$ & $\mathrm{H}$ & $\mathrm{H}$ & $\mathrm{H}$ & $\mathrm{H}$ & $23 c$ & $3 c$ & $\mathrm{H}$ & $\mathrm{H}$ & ;1 & $\mathrm{H}$ \\
\hline RL6064 & 24 & $; 1^{-}$ & ; & ; & ; & $; 1^{-}$ & ; & 0 & ; & $; 1^{-}$ & ; & ; & $; 1^{-}$ & $; 1^{-}$ & $\mathrm{H}$ & ; & ; \\
\hline Transec & 25 & 0 & ; & ; & 0 & ; & 0 & 0 & 0 & 0 & 0 & 0 & 0 & ; & 0 & 0 & ; \\
\hline RL6078 & 26 & 1 & 1 & ; & 0 & $\mathrm{H}$ & $\mathrm{H}$ & $\mathrm{H}$ & $\mathrm{H}$ & $\mathrm{H}$ & ; & ; & $\mathrm{H}$ & $\mathrm{H}$ & $\mathrm{H}$ & $\mathrm{H}$ & $\mathrm{H}$ \\
\hline Gatcher & $10,27+31$ & ;1 & ;1 & ; 1 & $\mathrm{XX}^{+}$ & $\mathrm{H}$ & $\mathrm{X}$ & $X$ & $X^{-}$ & $\mathrm{X}$ & H & $\mathrm{H}$ & $X$ & $X^{-}$ & $\mathrm{H}$ & $\mathrm{H}$ & $\mathrm{H}$ \\
\hline CS2D-2M & 28 & 0 & 0 & 0 & 0 & 0 & $\mathrm{H}$ & $\mathrm{H}$ & $\mathrm{H}$ & $\mathrm{H}$ & $\mathrm{H}$ & $\mathrm{H}$ & $\mathrm{H}$ & $\mathrm{H}$ & $\mathrm{H}$ & 0 & ; \\
\hline RL6080 & 29 & 1 & ; $1^{-}$ & ; & ; & ; 1 & 1 & $; 12^{-}$ & 12 & ;1 & 1 & $; 1^{-}$ & ;1 & $; 1^{-}$ & $; 1^{-}$ & $; 1$ & 12 \\
\hline RL6049 & 30 & $23 c$ & $22^{+}$ & $12^{-}$ & $23^{-}$ & $12^{-}$ & $23 c$ & $3 c 3$ & $23^{-}$ & $23 \mathrm{c}$ & $22^{+}$ & $23^{-}$ & $12^{-}$ & $23^{-}$ & $23^{-}$ & 12 & 12 \\
\hline RL5497 & 32 & $22^{+}$ & 2 & 12 & $22^{+}$ & 12 & 12 & $23 c$ & $23^{-}$ & 12 & $22^{+}$ & 12 & 12 & 12 & 12 & $1+2$ & 12 \\
\hline RL6057 & 33 & $3^{-}$ & $23^{-}$ & $3^{-}$ & $3^{-}$ & $23 \mathrm{C}$ & $22^{+}$ & $3 \mathrm{C} 3$ & $3^{-}$ & $22^{+}$ & $2+3 c$ & $23^{-}$ & $22^{+}$ & $3^{-}$ & $2^{+} 3^{-}$ & $3^{-}$ & $3^{-}$ \\
\hline RL6058 & 34 & $\mathrm{H}$ & $3-3$ & $3-3$ & $\mathrm{H}$ & $\mathrm{H}$ & $\mathrm{H}$ & $\mathrm{H}$ & $\mathrm{H}$ & $\mathrm{H}$ & $\mathrm{H}$ & $\mathrm{H}$ & $\mathrm{H}$ & $\mathrm{H}$ & $\mathrm{H}$ & $\mathrm{H}$ & $\mathrm{H}$ \\
\hline RL5711 & 35 & $\mathrm{H}$ & $\mathrm{H}$ & $\mathrm{H}$ & $\mathrm{H}$ & $\mathrm{H}$ & $\mathrm{H}$ & $\mathrm{H}$ & $\mathrm{H}$ & $\mathrm{H}$ & $\mathrm{H}$ & $\mathrm{H}$ & $\mathrm{H}$ & $\mathrm{H}$ & $\mathrm{H}$ & $\mathrm{H}$ & $\mathrm{H}$ \\
\hline
\end{tabular}

a Infection types follow 0-4 scale (22): $0=$ no uredinia or other macroscopic signs of infection; ; = no uredinia, but hypersensitive necrotic or chlorotic flecks of varying size; $1=$ small uredinia surrounded by necrosis; $2=$ small to medium uredinia surrounded by green islands; $X=$ random distribution of variable-sized uredinia on single leaf with a pure culture; $3,4(=\mathrm{H})=$ medium to large uredinia without chlorosis or necrosis; $+=$ uredinia somewhat larger than normal for the infection type; $=$ uredinia somewhat smaller than normal for infection type; $\mathrm{c}=$ more chlorosis than normal for infection type. More than one designation $=$ range of infection types.

b 9-day-old seedlings inoculated.

c 14-day-old seedlings inoculated.

d High; infection type 3 or higher.

Table 4. Seedling infection types displayed by 61 Chinese spring wheats when tested with 14 races of Puccinia triticina and postulated $L r$ genes

\begin{tabular}{|c|c|c|c|c|c|c|c|c|c|c|c|c|c|c|c|c|c|}
\hline \multirow[b]{2}{*}{ Cultivar $^{\mathrm{b}}$} & \multicolumn{16}{|c|}{ P. triticina race and infection type ${ }^{\mathrm{a}}$} & \multirow[b]{2}{*}{$\begin{array}{c}\text { Postulated } \\
L r \text { genes }\end{array}$} \\
\hline & $\begin{array}{c}\text { BBB/ } \\
\text { BB }\end{array}$ & $\begin{array}{c}\text { CBJ/ } \\
\text { QB }\end{array}$ & $\begin{array}{c}\text { CBJ/ } \\
\text { QL }\end{array}$ & $\begin{array}{c}\text { CBJ/ } \\
\text { QQ }\end{array}$ & $\begin{array}{c}\text { CCJ/ } \\
\text { SP }\end{array}$ & $\begin{array}{l}\mathbf{L C J} / \\
\mathbf{B N}^{\mathbf{c}}\end{array}$ & $\begin{array}{l}\text { LCJ/ } \\
\text { BNd }^{d}\end{array}$ & $\begin{array}{c}\mathbf{N C J} / \\
\mathbf{B N}^{\mathbf{c}}\end{array}$ & $\begin{array}{l}\mathbf{N C J} / \\
\mathbf{B N}^{\mathbf{d}}\end{array}$ & $\begin{array}{c}\text { TBB/ } \\
\text { JP }\end{array}$ & $\begin{array}{c}\text { TBD/ } \\
\text { TM }\end{array}$ & $\begin{array}{c}\text { TCB} / \\
\text { TB }\end{array}$ & $\begin{array}{c}\text { TCB/ } \\
\text { TD }\end{array}$ & $\begin{array}{c}\text { MFB/ } \\
\text { SP }\end{array}$ & $\begin{array}{c}\text { MCJ/ } \\
\text { QM }\end{array}$ & $\begin{array}{c}\text { MCJ/ } \\
\text { SP }\end{array}$ & \\
\hline 1 to 11 & $\mathrm{H}^{\mathrm{e}}$ & $\mathrm{H}$ & $\mathrm{H}$ & $\mathrm{H}$ & $\mathrm{H}$ & $\mathrm{H}$ & $\mathrm{H}$ & $\mathrm{H}$ & $\mathrm{H}$ & $\mathrm{H}$ & $\mathrm{H}$ & $\mathrm{H}$ & $\mathrm{H}$ & $\mathrm{H}$ & $\mathrm{H}$ & $\mathrm{H}$ & None \\
\hline & 0 & 0 & 0 & 0 & 0 & $\mathrm{H}$ & $\mathrm{H}$ & $\mathrm{H}$ & $\mathrm{H}$ & $\mathrm{H}$ & $\mathrm{H}$ & $\mathrm{H}$ & $\mathrm{H}$ & $\mathrm{H}$ & $\mathrm{H}$ & $\mathrm{H}$ & 1 \\
\hline 13 to 14 & & $\mathrm{H}$ & $\mathrm{H}$ & $\mathrm{H}$ & $\mathrm{H}$ & & & & & $\mathrm{H}$ & $\mathrm{H}$ & $\mathrm{H}$ & $\mathrm{H}$ & $\mathrm{H}$ & $\mathrm{H}$ & $\mathrm{H}$ & $3 b g$ \\
\hline 15 & ;1 & $; 12^{-}$ & $\mathrm{H}$ & $\mathrm{H}$ & $\mathrm{H}$ & H & $\stackrel{\leftrightarrow}{\mathrm{H}}$ & $\stackrel{H}{H}$ & H & $\mathrm{H}$ & $\mathrm{H}$ & & $; 1=$ & $\mathrm{H}$ & $\mathrm{H}$ & $\mathrm{H}$ & 10 \\
\hline 16 to 31 & 1 & $; 1$ & $1+3 c$ & 1 & $1^{+}$ & 1 & $1^{+}$ & 1 & 1 & $1^{+}$ & $1^{+}$ & 1 & $1^{+}$ & 1 & $1^{+}$ & $1^{+}$ & 16 \\
\hline 32 to 36 & $13 c$ & $; 1^{-}$ & 0 & 0 & $\mathrm{H}$ & $\mathrm{H}$ & $\mathrm{H}$ & $\mathrm{H}$ & $\mathrm{H}$ & ; & ; & $\mathrm{H}$ & $\mathrm{H}$ & $\mathrm{H}$ & $\mathrm{H}$ & $\mathrm{H}$ & 26 \\
\hline 37 & ; & $; 1^{-}$ & ; & 0 & $23 c$ & $; 1$ & $; 1^{-}$ & ; & ; & ; & ; & $\mathrm{H}$ & $\mathrm{H}$ & $\mathrm{H}$ & $3 c$ & $23 \mathrm{c}$ & 3,26 \\
\hline 38 to 40 & r & $-{ }^{\mathrm{f}}$ & - & 0 & $22+$ & ; & $; 1^{-}$ & ; & ; & ; & ; & $\mathrm{H}$ & $\mathrm{H}$ & $\mathrm{H}$ & $3 c$ & $23 c$ & 3,26 \\
\hline 41 to 44 & $; 1^{-}$ & $; 1^{-}$ & ; & 0 & 1 & 1 & $11^{+}$ & 1 & $1^{+}$ & ; & ; & 1 & $; 1$ & 1 & $1^{+}$ & $1^{+}$ & 16,26 \\
\hline 45 & ; & $; 1=$ & ; & 0 & $\mathrm{H}$ & $\mathrm{H}$ & $\mathrm{H}$ & $\mathrm{H}$ & $\mathrm{H}$ & ; & ; & $2+3$ & H & $3^{-}$ & $3 c$ & $\mathrm{H}$ & $26,+^{\mathrm{g}}$ \\
\hline 46 & 1 & $; 1^{-}$ & ; & 0 & $\mathrm{H}$ & $\mathrm{H}$ & $\mathrm{H}$ & $\mathrm{H}$ & $\mathrm{H}$ & ; & ; & $\mathrm{H}$ & $\mathrm{H}$ & $2+3 c$ & $\mathrm{H}$ & $\mathrm{H}$ & $26,+$ \\
\hline 47 & 1 & $; 1$ & ; & 0 & $3 c 3$ & $\mathrm{H}$ & $23 c$ & $3 \mathrm{c} 3$ & $2+3$ & ; & ; & $23 c$ & $\mathrm{H}$ & $\mathrm{H}$ & $3 \mathrm{c} 3$ & $3 \mathrm{c} 3$ & $26,+$ \\
\hline 48 & 1 & 1 & ; & 0 & $3 \mathrm{c} 3$ & $3 c$ & $2^{+} 3 c$ & $23 c$ & $3 \mathrm{c} 3$ & ; & ; & $23 c$ & $2^{+} 3$ & $1^{+} 2$ & $1^{+} 3 \mathrm{c}$ & $2+3 c$ & $26,+$ \\
\hline 49 & 1 & - & - & 0 & $2^{+} 3 c$ & $2+3 c$ & $2^{+} \mathrm{c}$ & $\mathrm{H}$ & $3 \mathrm{c} 3$ & ; & ; & $22^{+} \mathrm{c}$ & $\mathrm{H}$ & $1^{+}$ & $2^{+} 3 c$ & $\mathrm{H}$ & $26,+$ \\
\hline 50 & . & - & - & 0 & 0 & $\mathrm{H}$ & $3 \mathrm{c} 3$ & $2^{+}$ & $\mathrm{H}$ & ; & ; & 12 & $2^{+} 3$ & $2^{+}$ & $2^{+} 3 \mathrm{c}$ & $23 \mathrm{c}$ & $1,26,+$ \\
\hline 51 to 58 & ; & - & - & 0 & $1^{+}$ & 1 & $1^{+}$ & $1^{+}$ & $1^{+}$ & ; & ; & $11^{+}$ & 1 & 1 & & $1^{+}$ & $16,23,26$ \\
\hline 59 & 3-3 & $; 23^{-}$ & $12^{-}$ & $3 \mathrm{c}$ & $2+3$ & $3^{-c}$ & $2+3 c$ & $1^{+}$ & 12 & 2 & 1 & $3 \mathrm{c} 3$ & $3 c$ & $22^{+}$ & $22^{+}$ & $2^{+}$ & + \\
\hline 60 & $\mathrm{H}$ & 1 & $\mathrm{X}$ & $; 1^{-}$ & $\mathrm{H}$ & $\mathrm{H}$ & $\mathrm{H}$ & $\mathrm{H}$ & $\mathrm{H}$ & $\mathrm{H}$ & $\mathrm{H}$ & $\mathrm{H}$ & $\mathrm{H}$ & $\mathrm{H}$ & $\mathrm{H}$ & $\mathrm{H}$ & + \\
\hline 61 & $\mathrm{H}$ & $; 1$ & $1+3 c$ & 1 & $\mathrm{H}$ & $\mathrm{H}$ & $\mathrm{H}$ & $\mathrm{H}$ & $\mathrm{H}$ & $\mathrm{H}$ & $\mathrm{H}$ & $\mathrm{H}$ & $\mathrm{H}$ & $\mathrm{H}$ & $\mathrm{H}$ & $\mathrm{H}$ & + \\
\hline
\end{tabular}

${ }^{a}$ Infection types follow $0-4$ scale (22): $0=$ no uredinia or other macroscopic signs of infection; ; = no uredinia, but hypersensitive necrotic or chlorotic flecks of varying size; 1 = small uredinia surrounded by necrosis; $2=$ small to medium uredinia surrounded by green islands; $\mathrm{X}=$ random distribution of variable-sized uredinia on single leaf with a pure culture; $3,4(=\mathrm{H})=$ medium to large uredinia without chlorosis or necrosis; $+=$ uredinia somewhat larger than normal for infection type; $-=$ uredinia somewhat smaller than normal for infection type; $\mathrm{c}=$ more chlorosis than normal for infection type. More than one designation $=$ range of infection types

${ }^{\mathrm{b}}$ Numbers of cultivars refer to those given in Table 1 .

c 9-day-old seedlings inoculated.

d 14-day-old seedlings inoculated.

e High; infection type 3 or higher.

f Data not available.

$\mathrm{g}+$ indicates presence of an unidentified resistance gene. 
some winter wheats appears to be European wheat cultivars Lovrin 10, Lovrin 13, Predgornaia 2, Rusalka, Kavkaz, etc. (Tables 1 and 2) (10). There is a problem in the pedigree of spring wheat Guinong 93-9, supposedly derived from an intergeneric cross involving a durum wheat, Haynaldia villosa, and Avena fatua (Table 1 ). None of the three parents could have contributed the wheat-rye translocation, but it is nevertheless present in Guinong 93-9. Similarly, pedigrees of all other wheats (numbered 38-40 and 51-58, Table 1) are doubtful because each cultivar carries the 1B.1R translocation (Table 4).

Gene $L r l$ was present in combination with $\operatorname{Lr} 13$ in Tianshui 872, with $\operatorname{Lr} 26$ in Yumai 13, and with an unidentified gene in Bainong 3217, Bainong 64, and Ji 89-6232 (Table 5). Postulation of the Lrl-Lrl3 combination was based on the low IT 0; of Tianshui with Lrl avirulent races $\mathrm{BBB} / \mathrm{BN}, \mathrm{CBJ} / \mathrm{QB}, \mathrm{CBJ} / \mathrm{QL}, \mathrm{CBJ} / \mathrm{QQ}$, and
$\mathrm{CCJ} / \mathrm{SP}$; IT $\mathrm{X}$ or $3 \mathrm{c}$ with $\operatorname{Lr} 13$ avirulent races $\mathrm{LCJ} / \mathrm{BN}$ and $\mathrm{NCJ} / \mathrm{BN}$; and high ITs with the remaining races that carried virulences for $\mathrm{Lrl}$ and Lr13. Postulations of other combinations involved similar criteria. The unknown gene (or genes) involved in combination with $\mathrm{Lrl}$ conferred ITs in the 2 to $2^{+} 3$ range, and it appears that virulence was absent for gene(s) present in at least Bainong 64 and Ji 89-6232. Among other gene combinations, the most frequent involved $L r 3$ and $L r 26$ in four spring (37 to 40, Table 4) and two facultative/winter wheats (Jinhua 5 and Siyang 128, Table 5); Lr16 and Lr26 in four spring (41 to 44, Table 4) and seven facultative/winter wheats (70 to 76, Table 5); and Lr26 and an unidentified resistance gene in five spring (45 to 49, Table 4) and eight facultative/winter wheats ( 78 to 85 , Table 5 ).

Eight spring wheats (51 to 58, Table 4), all supposedly derived from a $T$. $d u$ rum/Haynaldia villosa cross, carried genes
Lr16, Lr23, and Lr26. This indicated that the pedigree is incorrect. It is likely that a Lr23-carrying durum was crossed with a bread wheat carrying Lr16 and Lr26. Combinations involving three genes were also present in eight winter wheats (Table 5 ). Low infection types of three spring (59 to 61, Table 4) and nine facultative/winter wheats (94 to 102, Table 5) could not be associated with any known resistance gene. This included winter wheat Zhong 4, which is known to carry seven pairs of chromosomes from Agropyron intermedium. Lack of virulence for some of these genes indicates their likely usefulness in reducing losses caused by leaf rust.

Evaluation of 28 spring wheats at two field locations indicated that about half of them carried good to moderate levels of resistance under heavy rust pressure (Table 6). In general, cultivars displayed similar ratings at both locations. Although six cultivars, Chuanyu 5, Xuzhou 21, Zhejiang

Table 5. Seedling infection types displayed by 102 Chinese facultative and winter wheats when inoculated with 14 races of Puccinia triticina and postulated $L r$ genes

\begin{tabular}{|c|c|c|c|c|c|c|c|c|c|c|c|c|c|c|c|c|c|}
\hline \multirow[b]{2}{*}{ Cultivar $^{\mathbf{b}}$} & \multicolumn{16}{|c|}{ P. triticina race and infection type ${ }^{a}$} & \multirow[b]{2}{*}{$\begin{array}{l}\text { Postulated } \\
L r \text { genes }\end{array}$} \\
\hline & $\begin{array}{c}\text { BBB/ } \\
\text { BB }\end{array}$ & $\begin{array}{c}\text { CBJ/ } \\
\text { QB }\end{array}$ & $\begin{array}{c}\text { CBJ/ } \\
\text { QL }\end{array}$ & $\begin{array}{c}\text { CBJ/ } \\
\text { QQ }\end{array}$ & $\begin{array}{c}\text { CCJ/ } \\
\text { SP }\end{array}$ & $\begin{array}{l}\mathbf{L C J} / \\
\mathrm{BN}^{\mathrm{c}}\end{array}$ & $\begin{array}{l}\mathbf{L C J} / \\
\mathbf{B N}^{\mathbf{d}}\end{array}$ & $\begin{array}{c}\text { NCJ/ } \\
\text { BNc }^{\mathbf{c}}\end{array}$ & $\begin{array}{l}\text { NCJ/ } \\
\text { BN }^{\mathbf{d}}\end{array}$ & $\begin{array}{c}\text { TBB/ } \\
\text { JP }\end{array}$ & $\begin{array}{c}\text { TBD/ } \\
\text { TM }\end{array}$ & $\begin{array}{c}\text { TCB/ } \\
\text { TB }\end{array}$ & $\begin{array}{c}\text { TCB/ } \\
\text { TD }\end{array}$ & $\begin{array}{c}\text { MFB/ } \\
\text { SP }\end{array}$ & $\begin{array}{c}\text { MCJ/ } \\
\text { QM }\end{array}$ & $\begin{array}{c}\text { MCJ/ } \\
\text { SP }\end{array}$ & \\
\hline 1 to 18 & $\mathrm{H}^{\mathrm{e}}$ & $\mathrm{H}$ & $\mathrm{H}$ & $\mathrm{H}$ & $\mathrm{H}$ & $\mathrm{H}$ & $\mathrm{H}$ & $\mathrm{H}$ & $\mathrm{H}$ & $\mathrm{H}$ & $\mathrm{H}$ & $\mathrm{H}$ & $\mathrm{H}$ & $\mathrm{H}$ & $\mathrm{H}$ & $\mathrm{H}$ & None \\
\hline 19 to 22 & 0 & $-^{\mathrm{f}}$ & - & 0 & 0 & $\mathrm{H}$ & $\mathrm{H}$ & $\mathrm{H}$ & $\mathrm{H}$ & $\mathrm{H}$ & $\mathrm{H}$ & $\mathrm{H}$ & $\mathrm{H}$ & $\mathrm{H}$ & $\mathrm{H}$ & $\mathrm{H}$ & 1 \\
\hline 23 to 24 & $3 c$ & $\mathrm{H}$ & $\mathrm{H}$ & $\mathrm{H}$ & $\mathrm{H}$ & $X$ & X & $X$ & X & $\mathrm{H}$ & $\mathrm{H}$ & $\mathrm{H}$ & $\mathrm{H}$ & $\mathrm{H}$ & $\mathrm{H}$ & $\mathrm{H}$ & 13 \\
\hline 25 & $3 c$ & $\mathrm{H}$ & $\mathrm{H}$ & $\mathrm{H}$ & $\mathrm{H}$ & $\mathrm{H}$ & $\mathrm{H}$ & $\mathrm{H}$ & $\mathrm{H}$ & $\mathrm{H}$ & $\mathrm{H}$ & $\mathrm{H}$ & $\mathrm{H}$ & $\mathrm{H}$ & $\mathrm{H}$ & $\mathrm{H}$ & $14 a$ \\
\hline 26 to 34 & $3 \mathrm{c} 3$ & $1^{+}$ & 1 & 1 & $1^{+} 3 \mathrm{c}$ & 1 & $1^{+}$ & $1^{+}$ & $1^{+}$ & $1^{+}$ & $1^{+}$ & $1^{+}$ & $1^{+}$ & $1^{+}$ & $1^{+} 2$ & $1^{+} 3 \mathrm{c}$ & 16 \\
\hline 35 to 61 & 1 & 1 & ; & 0 & $\mathrm{H}$ & $\mathrm{H}$ & $\mathrm{H}$ & $\mathrm{H}$ & $\mathrm{H}$ & ; & ; & $\mathrm{H}$ & $\mathrm{H}$ & $\mathrm{H}$ & $\mathrm{H}$ & $\mathrm{H}$ & 26 \\
\hline 62 & 0 & 0 & 0 & 0 & 0 & $3 c$ & X & X & $\mathrm{X}$ & $\mathrm{H}$ & $\mathrm{H}$ & $\mathrm{H}$ & $\mathrm{H}$ & $\mathrm{H}$ & $\mathrm{H}$ & $\mathrm{H}$ & 1,13 \\
\hline 63 & 0 & 0 & 0 & 0 & 0 & $\mathrm{H}$ & $\mathrm{H}$ & $\mathrm{H}$ & $\mathrm{H}$ & ; & ; & $\mathrm{H}$ & $\mathrm{H}$ & $\mathrm{H}$ & $\mathrm{H}$ & $\mathrm{H}$ & 1,26 \\
\hline 64 & 0 & 0 & 0 & 0 & 0 & $\mathrm{H}$ & $\mathrm{H}$ & $\mathrm{H}$ & $2+3 c$ & $2+3 c$ & $22^{+}$ & $2+3 c$ & $\mathrm{H}$ & $\mathrm{H}$ & $\mathrm{H}$ & $2^{+} 3$ & $1,+^{\mathrm{g}}$ \\
\hline 65 to 66 & 0 & 0 & 0 & 0 & 0 & $2+3 c$ & $2^{+}$ & $2^{+}$ & $2+3$ & $22^{+}$ & 2 & $22^{+}$ & $2^{+}$ & 2 & $22^{+}$ & $2+3$ & $1,+$ \\
\hline 67 to 68 & ; & 1 & ; & ; & $22^{+}$ & $; 1^{-}$ & $; 1$ & ; & ; & ; & ; & $\mathrm{H}$ & $\mathrm{H}$ & $\mathrm{H}$ & 12 & $3-3$ & 3,26 \\
\hline 69 & ; & - & - & $23^{-}$ & 12 & $; 1$ & $; 1$ & ; & $; 1^{-}$ & $23^{-}$ & $\mathrm{H}$ & $\mathrm{H}$ & $\mathrm{H}$ & 12 & $3^{-}$ & $3^{-}$ & $3,+$ \\
\hline 70 to 76 & 1 & $1^{+}$ & 1 & 0 & $1^{+} 3 c$ & 1 & 1 & $1^{+}$ & $1^{+}$ & ; & ; & $1^{+}$ & $1^{+}$ & $1^{+}$ & 12 & $3 c$ & 16,26 \\
\hline 77 & ; & $1^{+}$ & 1 & 0 & $1^{+}$ & 1 & $1^{+}$ & $1^{+}$ & $1^{+}$ & $1^{+}$ & $1^{+}$ & $1^{+}$ & $1^{+}$ & $1^{+}$ & 12 & $1^{+}$ & $16,+$ \\
\hline 78 to 79 & 1 & 1 & ; & 0 & $\mathrm{H}$ & $\mathrm{H}$ & $\mathrm{H}$ & $\mathrm{H}$ & $\mathrm{H}$ & ; & ; & $\mathrm{H}$ & $\mathrm{H}$ & $2^{+}$ & $\mathrm{H}$ & $\mathrm{H}$ & $26,+$ \\
\hline 80 & 1 & - & - & 0 & $2^{+} 3$ & $\mathrm{H}$ & $\mathrm{H}$ & $\mathrm{H}$ & $3 c 3$ & ; & ; & $\mathrm{H}$ & $\mathrm{H}$ & 2 & $3 c$ & $3 c 3$ & $26,+$ \\
\hline 81 & 1 & - & - & 0 & - & $\mathrm{H}$ & $\mathrm{H}$ & $\mathrm{H}$ & $\mathrm{H}$ & ; & ; & $\mathrm{H}$ & $\mathrm{H}$ & $2^{+} 3$ & $\mathrm{H}$ & $\mathrm{H}$ & $26,+$ \\
\hline 82 & 1 & 1 & ; & 0 & $3 \mathrm{c} 3$ & $1^{+}$ & $22^{+} \mathrm{c}$ & $1^{+} 2^{+}$ & $2+3$ & ; & ; & $2+3 c$ & $2+3$ & $\mathrm{H}$ & $1+2$ & $\mathrm{H}$ & $26,+$ \\
\hline 83 & 1 & - & - & 0 & $\mathrm{H}$ & $22^{+}$ & $2^{+}$ & $\mathrm{H}$ & $3 c$ & ; & 0 & $\mathrm{H}$ & $\mathrm{H}$ & $2^{+} 3$ & $2^{+} 3$ & $\mathrm{H}$ & $26,+$ \\
\hline 84 & 1 & - & - & 0 & $2+3 c$ & $\mathrm{H}$ & $\mathrm{H}$ & $\mathrm{H}$ & $\mathrm{H}$ & ; & ; & $\mathrm{H}$ & $\mathrm{H}$ & $1^{+}$ & $\mathrm{H}$ & $\mathrm{H}$ & $26,+$ \\
\hline 85 & 1 & - & - & 0 & $2+3 c$ & $2^{+}$ & $2^{+}$ & $\mathrm{H}$ & $\mathrm{H}$ & ; & ; & $\mathrm{H}$ & $\mathrm{H}$ & $2+3$ & $3 c$ & $\mathrm{H}$ & $26,+$ \\
\hline 86 & 0 & - & - & 0 & 0 & $\mathrm{X}$ & $\mathrm{X}^{+}$ & $\mathrm{X}$ & $\mathrm{X}^{+}$ & ; & ; & $\mathrm{H}$ & $\mathrm{H}$ & $\mathrm{H}$ & $\mathrm{H}$ & $\mathrm{H}$ & $1,13,26$ \\
\hline 87 & - & - & - & 0 & 0 & 0 & $\mathrm{X}$ & $; 1$ & $\mathrm{H}$ & ; & ; & 2 & $\mathrm{X}^{-}$ & $1^{+} 3 \mathrm{c}$ & 12 & ; & $1,26,+$ \\
\hline 88 to 90 & ; & ; & $1^{-}$ & 0 & $; 1^{-}$ & ; & ; & 0 & ; & ; & 0 & 12 & 12 & $1^{+}$ & 12 & $; 1^{-}$ & $3,16,26$ \\
\hline 91 to 92 & 0 & - & - & 0 & $; 1^{-}$ & $; 1^{-}$ & $; 12^{-}$ & ; & ; & ; & ; & $3 c$ & $23 c$ & $3 c$ & $23 c$ & ;12 & $3,26,+$ \\
\hline 93 & - & - & - & 0 & $; 1$ & $1^{+}$ & $; 1^{-}$ & ; & ; & ; & ; & $1^{+}$ & $1^{+}$ & $; 1$ & $; 1$ & ;12 & $16,23,26$ \\
\hline 94 & $3 c$ & $\mathrm{H}$ & $\mathrm{H}$ & $3 \mathrm{c} 3$ & $\mathrm{H}$ & $3 c$ & $\mathrm{H}$ & $\mathrm{H}$ & $\mathrm{H}$ & $2+3 c$ & $22^{+}$ & $\mathrm{H}$ & $\mathrm{H}$ & $\mathrm{H}$ & $22^{+}$ & $3 \mathrm{c} 3$ & + \\
\hline 95 & $\mathrm{H}$ & - & - & $\mathrm{H}$ & $\mathrm{H}$ & $\mathrm{H}$ & $\mathrm{H}$ & $\mathrm{H}$ & $\mathrm{H}$ & $\mathrm{H}$ & $\mathrm{H}$ & $2^{+} 3$ & $\mathrm{H}$ & $22^{+}$ & $\mathrm{H}$ & $2+3$ & + \\
\hline 96 & $\mathrm{H}$ & - & - & $\mathrm{H}$ & $3 \mathrm{c} 3$ & $\mathrm{H}$ & $\mathrm{H}$ & $\mathrm{H}$ & $\mathrm{H}$ & $\mathrm{H}$ & $22^{+}$ & $\mathrm{H}$ & $\mathrm{H}$ & $22^{+}$ & $3 \mathrm{c} 3$ & $3 \mathrm{c} 3$ & + \\
\hline 97 & $2^{+} 3$ & - & - & $\mathrm{H}$ & $\mathrm{H}$ & $\mathrm{H}$ & $\mathrm{H}$ & $\mathrm{H}$ & $2+3$ & $22^{+}$ & 2 & $\mathrm{H}$ & $\mathrm{H}$ & $\mathrm{H}$ & $\mathrm{H}$ & $\mathrm{H}$ & + \\
\hline 98 to 100 & $23^{-}$ & - & - & $23^{-}$ & 3-3 & $23^{-}$ & $3^{-}$ & $3-3$ & $3 c 3$ & 12 & 12 & $2^{+} 3$ & $3 \mathrm{c} 3$ & 12 & $3-3$ & $3-3$ & + \\
\hline 101 & 0 & - & - & $; 1^{-}$ & 0 & 0 & 0 & ; & 0 & 0 & 0 & ; & ; & 0 & 0 & ; & + \\
\hline 102 & $2+3 c$ & - & - & $2^{+} 3$ & $3 \mathrm{c} 3$ & $2^{+}$ & $22^{+}$ & $\mathrm{H}$ & $\mathrm{H}$ & $2^{+} 3$ & 2 & $2^{+}$ & $\mathrm{H}$ & $2^{+}$ & $2^{+} 3$ & $\mathrm{H}$ & + \\
\hline
\end{tabular}

${ }^{a}$ Infection types follow a 0 to 4 scale (22), where $0=$ no uredinia or other macroscopic signs of infection; ; = no uredinia, but hypersensitive necrotic or chlorotic flecks of varying size present; $1=$ small uredinia surrounded by necrosis; $2=$ small to medium uredinia surrounded by green islands; $X=$ random distribution of variable-sized uredinia on single leaf with a pure culture; 3 and $4(=\mathrm{H})=$ medium to large uredinia without chlorosis or necrosis; $+=$ uredinia somewhat larger than normal for the infection type; $-=$ uredinia somewhat smaller than normal for the infection type; $\mathrm{c}=$ more chlorosis than normal for the infection type. More than one designation represents a range of infection types.

b Numbers of cultivars refer to those given in Table 2.

c 9-day-old seedlings inoculated.

d 14-day-old seedlings inoculated.

${ }^{\mathrm{e}}$ High; infection type 3 or higher.

$f$ Data not available.

$\mathrm{g}+$ indicates presence of an unidentified resistance gene. 
4, Fujing 5114, Wuhan 3, and Zhengzhou 871, displayed high seedling reactions with at least one of the two races, their resistance in adult plant tests indicated the presence of effective adult plant resistance genes. It is also interesting to note that cultivars that displayed low seedling infection type due to the presence of Lr16 were not always resistant in the field tests. The most susceptible and the most resistant cultivars with Lr16, Mianyang 11 and Yangmai 87-158, displayed 100S and 10MSS responses, respectively; responses of other cultivars fell somewhere in between. This is in agreement with earlier results (19) that showed that although $\operatorname{Lr} 16$ is effective at the seedling stage, it does not confer much resistance in adult plants. Therefore, additional genes for adult plant resistance must be present in some of these wheats with $\operatorname{Lrl6}$.

Gene Lr34, known to confer slow rusting resistance to leaf rust (18), may be present in at least seven cultivars, Xuzhou 21, Shanghai 3, Shanghai 5, Chuanmai 18, Fujing 5114, Wuhan 3, and New Kehan 9. These cultivars displayed clear symptoms of leaf tip necrosis, known to be linked to Lr34 (15). The presence of Lr34 in Chinese wheats is not surprising, as Dyck (6) has shown its presence in Chinese Spring wheat. Other cultivars that do not show leaf tip necrosis clearly may still carry $L r 34$, as the symptoms of necrosis can be modified in some genetic backgrounds. The slow rusting resistance (based on Lr34) of these cultivars should be of a durable nature $(7,21)$.

In conclusion, it can be stated that only limited genetic diversity was observed for the named genes involved in seedling resistance of Chinese wheat cultivars. In total, nine named genes, Lrl (in 13 cultivars), $\operatorname{Lr3}$ (12), $\operatorname{Lr3bg}$ (2), $\operatorname{LrlO}(1), \operatorname{Lrl3}$ (4), Lr14a (1), Lr16 (49), Lr23 (9), and Lr26 (81), were identified. These known

Table 6. Seedling reactions, postulated $L r$ genes, and adult plant leaf rust ratings at two field locations of 28 Chinese spring wheat cultivars when tested with two Puccinia triticina races

\begin{tabular}{|c|c|c|c|c|c|c|}
\hline \multirow[b]{2}{*}{ No.b } & \multirow[b]{2}{*}{ Cultivar } & \multicolumn{2}{|c|}{$\begin{array}{l}P . \text { triticina race } \\
\text { and seedling } \text { IT }^{\mathrm{a}}\end{array}$} & \multirow{2}{*}{$\begin{array}{l}\text { Postulated } \\
L r \text { genes }\end{array}$} & \multicolumn{2}{|c|}{ Field leaf rust ratingd } \\
\hline & & $\overline{\mathrm{TBD} / \mathrm{TM}}$ & TCB/TD & & Cd. Obregon & El Batan \\
\hline 9 & Chuanyu 5 & $\mathrm{H}$ & $\mathrm{H}$ & None & $40 \mathrm{~S}$ & 40MSS \\
\hline 10 & Chuanmai 22 & $\mathrm{H}$ & $\mathrm{H}$ & None & $100 \mathrm{~S}(\mathrm{~N})$ & $100 \mathrm{~S}(\mathrm{~N})$ \\
\hline 12 & Xuzhou 21 & $\mathrm{H}$ & $\mathrm{H}$ & None & 30MSS & 40MSS \\
\hline 14 & Ningxia 86Y1608 & $\mathrm{H}$ & $\mathrm{H}$ & Lr3bg & $100 \mathrm{~S}(\mathrm{~N})$ & $100 \mathrm{~S}(\mathrm{~N})$ \\
\hline 15 & Kefeng 3 & $\mathrm{H}$ & $; 1=$ & Lr10 & $100 \mathrm{~S}$ & $100 \mathrm{~S}(\mathrm{~N})$ \\
\hline 16 & Yangmai 4 & $1^{+}$ & $1^{+}$ & Lr16 & 40MSS & 30MSS \\
\hline 17 & Yangmai 5 & $1^{+}$ & $1^{+}$ & Lr16 & 20MSS & 10MSS \\
\hline 19 & Yangmai 85-85 & 1 & $1^{+}$ & Lr16 & 70MSS & 60MSS \\
\hline 20 & Yangmai 87-158 & 12 & $1^{+}$ & Lr16 & 10MSS & 10MSS \\
\hline 24 & Mianyang 11 & $1^{+}$ & $1^{+}$ & Lr16 & $100 \mathrm{~S}(\mathrm{~N})$ & $100 \mathrm{~S}$ \\
\hline 25 & Chuanyu 10 & 1 & $1^{+}$ & Lr16 & $80 \mathrm{~S}$ & 70MSS \\
\hline 26 & Kefeng 5 & $1^{+}$ & $1^{+}$ & Lr16 & 40MSS & 30MSS \\
\hline 27 & Kehan 10 & 1 & $; 1$ & Lr16 & 80MSS & 80MSS \\
\hline 28 & Shanghai 3 & $3 c 3$ & $1+3 c$ & Lr16 & 40MSS & 30MSS \\
\hline 29 & Shanghai 5 & $1^{+}$ & $1^{+}$ & Lr16 & 20MSS & 20MSS \\
\hline 32 & Zhejiang 4 & ; & $\mathrm{H}$ & $\operatorname{Lr} 26$ & 60MSS & $60 \mathrm{MSS}$ \\
\hline 33 & Yumai 5 & ; & $\mathrm{H}$ & $\operatorname{Lr} 26$ & $100 \mathrm{~S}(\mathrm{~N})$ & $100 \mathrm{~S}(\mathrm{~N})$ \\
\hline 34 & Yumai 7 & ; & $\mathrm{H}$ & $\operatorname{Lr} 26$ & $100 \mathrm{~S}(\mathrm{~N})$ & $100 \mathrm{~S}(\mathrm{~N})$ \\
\hline 35 & Yumai 15 & ; & $\mathrm{H}$ & $\operatorname{Lr} 26$ & $100 \mathrm{~S}(\mathrm{~N})$ & $100 \mathrm{~S}$ \\
\hline 37 & Chuanyu 11 & ; & $\mathrm{H}$ & Lr3,26 & $100 \mathrm{~S}$ & $100 \mathrm{~S}(\mathrm{~N})$ \\
\hline 41 & Chuanmai 18 & ; & $; 1$ & $\operatorname{Lr16,26}$ & 30MSS & 30MSS \\
\hline 45 & Fujing 5114 & ; & $\mathrm{H}$ & $\operatorname{Lr} 26,+$ & $5 \mathrm{MS}$ & $15 \mathrm{MS}$ \\
\hline 46 & Wuhan 3 & ; & $\mathrm{H}$ & $\operatorname{Lr} 26,+$ & $15 \mathrm{MS}$ & $15 \mathrm{MSS}$ \\
\hline 47 & Zhengzhou 871 & ; & $\mathrm{H}$ & $\operatorname{Lr} 26,+$ & 20MSS & 30MSS \\
\hline 48 & Zhengzhou 872 & ; & $2+3$ & $\operatorname{Lr} 26,+$ & $100 \mathrm{~S}(\mathrm{~N})$ & $100 \mathrm{~S}(\mathrm{~N})$ \\
\hline 59 & New Kehan 9 & 1 & $3 c$ & + & $5 \mathrm{MS}$ & $1 \mathrm{MS}$ \\
\hline 60 & Chuanyu 8 & $\mathrm{H}$ & $\mathrm{H}$ & + & $60 \mathrm{~S}$ & $80 \mathrm{~S}$ \\
\hline 61 & Chuanyu 12 & $\mathrm{H}$ & $\mathrm{H}$ & + & $100 \mathrm{~S}(\mathrm{~N})$ & $100 \mathrm{~S}(\mathrm{~N})$ \\
\hline
\end{tabular}

a Taken from Table 4 and based on the 0 to 4 scale (22), where $0=$ no uredinia or other macroscopic signs of infection; ; = no uredinia, but hypersensitive necrotic or chlorotic flecks of varying size present; 1 = small uredinia surrounded by necrosis; 2 = small to medium uredinia surrounded by green islands; $\mathrm{X}=$ random distribution of variable-sized uredinia on single leaf with a pure culture; 3 and $4(=\mathrm{H})=$ medium to large uredinia without chlorosis or necrosis; $+=$ uredinia somewhat larger than normal for the infection type; - = uredinia somewhat smaller than normal for the infection type; $\mathrm{c}=$ more chlorosis than normal for the infection type. More than one designation represents a range of infection types.

b Numbers of cultivars refer to those given in Table 1.

c Taken from Table 4.

d Although independent tests were carried out with races TBD/TM and TCB/TD, we reported only the higher ratings in the two tests at each location. Leaf rust ratings involve two components: percent disease severity based on the modified Cobb scale (11), and reaction type as described in Roelfs et al. (12). The reaction types are: $\mathrm{MS}=$ moderately susceptible, medium sized uredinia without chlorosis or necrosis; $\mathrm{S}=$ susceptible, large uredinia without chlorosis or necrosis; MSS = moderately susceptible to susceptible; $(\mathrm{N})=$ necrotic leaves following high leaf rust infection.

genes would be ineffective in many areas of the world. Thirty-two cultivars carried resistance that could not be attributed to any named gene. In most cases, the level of this resistance was slight, and resistance was expressed with only a few races. Zhong 4, a 56-chromosome line carrying all chromosomes of Agropyron intermedium, displayed very low seedling reactions with all races (entry 101, Table 5). However, this resistance will have to be transferred to a wheat chromosome before it can be of any use. The adult plant resistance conferred by either Lr34 or unknown slow rusting genes in the studied spring wheats should interest breeders, as such resistance is often durable $(3,13,21)$. Pedigrees of 12 cultivars, supposedly derived from intergeneric crosses, are incorrect. Small seed samples of the Chinese cultivars can be obtained from $\mathrm{Z}$. H. He.

\section{ACKNOWLEDGMENTS}

We thank A. McNab for the technical review of the manuscript.

\section{LITERATURE CITED}

1. Bai, G., and Shaner, G. 1994. Scab of wheat: Prospects for control. Plant Dis. 78:760-766.

2. Browder, L. E., and Eversmeyer, M. G. 1980. Sorting of Puccinia recondita: Triticum infection-type data sets toward the gene-forgene model. Phytopathology 70:666-670.

3. Caldwell, R. M. 1968. Breeding for general and/or specific plant disease resistance. Pages 263-272 in: Proc. Int. Wheat Genet. Symp. 3rd. K. W. Finlay and K. W. Shepherd, eds.

4. CIMMYT. 1989. 1987/1988 CIMMYT World Wheat Facts and Trends. The wheat revolution revisited: Recent trends and future challenges. CIMMYT, Mexico City.

5. CIMMYT. 1996. CIMMYT 1995/96 World Wheat Facts and Trends: Understanding global trends in the use of wheat diversity and international flows of wheat genetic resources. CIMMYT, Mexico City.

6. Dyck, P. L. 1991. Genetics of adult-plant leaf rust resistance in 'Chinese Spring' and 'Sturdy' wheats. Crop Sci. 31:309-311.

7. Dyck, P. L., and Samborski, D. J. 1982. The inheritance of resistance to Puccinia recondita in a group of common wheat cultivars. Can. J. Genet. Cytol. 24:273-283.

8. Knott, D. R., and Johnson, R. 1983. Some additional comments on sorting infection-type data sets. Phytopathology 73:514-515.

9. Long, D. L., and Kolmer, J. A. 1989. A North American system of nomenclature for Puccinia recondita f. sp. tritici. Phytopathology 79:525-529.

10. McIntosh, R. A., Hart, G. E., and Gale, M. D. 1995. Catalogue of gene symbols for wheat. Pages 1333-1500 in: Proc. Int. Wheat Genet. Symp. 8th. Z. S. Li and Z. Y. Xin, eds.

11. Peterson, R. F., Campbell, A. B., and Hannah, A. E. 1948. A diagrammatic scale for estimating rust intensity of leaves and stem of cereals. Can. J. Res. Sect. C. 26:496-500.

12. Roelfs, A. P., Singh, R. P., and Saari, E. E. 1992. Rust diseases of wheat: Concepts and methods of disease management. CIMMYT, Mexico City.

13. Shaner, G., and Finney, R. E. 1980. New sources of slow leaf rusting resistance in wheat. Phytopathology 70:1183-1186.

14. Singh, R. P. 1991. Pathogenicity variations of Puccinia recondita f. sp. tritici and $P$. graminis f. sp. tritici in wheat-growing areas 
of Mexico during 1988 and 1989. Plant Dis. 75:790-794.

15. Singh, R. P. 1992. Association between gene Lr34 for leaf rust resistance and leaf tip necrosis in wheat. Crop Sci. 32:874-878.

16. Singh, R. P. 1993. Resistance to leaf rust in 26 Mexican wheat cultivars. Crop Sci. 33:633637.

17. Singh, R. P., and Gupta, A. K. 1991. Genes for leaf rust resistance in Indian and Pakistani wheats tested with Mexican pathotypes of Puccinia recondita f. sp. tritici. Euphytica 57:27-36.
18. Singh, R. P., and Gupta, A. K. 1992. Expression of wheat leaf rust resistance gene Lr34 in seedlings and adult plants. Plant Dis. 76:489491.

19. Singh, R. P., and Huerta-Espino, J. 1995. Inheritance of seedling and adult plant resistance to leaf rust in wheat cultivars Ciano 79 and Papago 86. Plant Dis. 79:35-38.

20. Singh, R. P., and Rajaram, S. 1991. Resistance to Puccinia recondita f. sp. tritici in 50 Mexican bread wheat cultivars. Crop Sci. 31:1472-1479.

21. Singh, R. P., and Rajaram, S. 1992. Genetics of adult-plant resistance to leaf rust in 'Frontana' and three CIMMYT wheats. Genome 35:24-31.

22. Stakman, E. C., Stewart, D. M., and Loegering, W. Q. 1962. Identification of physiologic races of Puccinia graminis var. tritici. U.S Agric. Res. Serv. E-617(rev.).

23. Statler, G. D. 1984. Probable genes for leaf rust resistance in several hard red spring wheats. Crop Sci. 24:883-886.

24. Zadoks, J. C., Chang, T. T., and Konzak, C. F. 1974. A decimal code for the growth stages of cereals. Weed Res. 14:415-421. 